\title{
A Framework for Research in Gamified Mobile Guide Applications using Embodied Conversational Agents (ECAs)
}

\author{
Ioannis Doumanis ${ }^{1}$, Serengul Smith ${ }^{2}$ \\ ${ }^{1}$ University of Middlesex, The Burroughs Hendon, London, i.doumanis@mdx.ac.uk \\ ${ }^{2}$ University of Middlesex, The Burroughs Hendon, London, S.Smith@mdx.ac.uk
}

\begin{abstract}
Mobile Guides are mobile applications that provide players with local and locationbased services (LBS), such as navigation assistance, where and when they need them most. Advances in mobile technologies in recent years have enabled the gamification of these applications, opening up new opportunities to transfer education and culture through game play. However, adding traditional game elements such as PBLs (points, badges, and leaderboards) alone cannot ensure that the intended learning outcomes will be met, as the player's cognitive resources are shared between the application and the surrounding environment. This distribution of resources prevents players from easily immersing themselves into the educational scenario. Adding artificial conversational characters (ECAs) that simulate the social norms found in real-life human-to-human guide scenarios has the potential to address this problem and improve the player's experience and learning of cultural narratives [1]. Although significant progress has been made towards creating game-like mobile guides with ECAs ([2], [3]), there is still a lack of a unified framework that enables researchers and practitioners to investigate the potential effects of such applications to players and how to approach the concepts of player experience, cognitive accessibility and usability in this context. This paper presents a theoretically-well supported research framework consisted of four key components: differences in players, different features of the gamified task, aspects of how the ECA looks, sound or behaves and different mobile environments. Furthermore, it provides based on this framework a working definition of what player experience, cognitive accessibility and usability are in the context of game-like mobile guide applications. Finally, a synthesis of the results of six empirical studies conducted within this research framework is discussed and a series of design guidelines for the effective gamification of mobile guide applications using ECAs are presented. Results show that an ECA can positively affect the quality of the player's experience, but it did not elicit better player retention of cultural narratives and navigation of routes.
\end{abstract}

Keywords: Gamification, Mobile Guide Applications (MGA), Embodied Conversational Agents (ECAs), Player experience, Cognitive Accessibility, Usability

\section{Introduction}

Over the past few years the world has seen tremendous progress in wireless technology and mobile devices, with wireless networks becoming more pervasive and providing more bandwidth than ever before and mobile devices becoming progressively smaller and more compact. Although the latest generation of mobile devices (e.g., Apple iPad [4]), has brought significant improvements both in terms of hardware and interactive features, the user interface (UI) is still based on the graphical user interface (GUI) first introduced in desktop environments. The improved hardware features have enabled designers to create cleaner UIs, but the new multi-touch style of interaction has forced them to ask for more compact information architectures (IA). The limited screen size and the size of the human fingertip make it difficult for users to swipe and touch screen icons, etc. 
This means that the GUI elements (and thereby the underlying IA) should support the completion of tasks in a limited number of user actions. Without IAs that minimize the user's actions and maximize the system output, the supporting GUIs become unnecessarily complicated. To address the above limitation, a number of mobile systems already offer information aggregation services. This means that the mobile system aggregates content from multiple resources on the World Wide Web (WWW) and pushes them to the user, formatted in a single presentation medium, with minimal user intervention. For example, in Siri [5] participants with the click-of-a-button can query in natural language information from multiple web resources simultaneously. To aid the user Siri provides categories of common queries that it understands. The output is usually formatted in short natural language sentences augmented with multimedia content (e.g., text and videos). This simplified IA (supported by natural language processing) enables collaborative completion of tasks as easily as working with another human being. In recent years, mobile designers in an effort to make mobile applications more playful and engaging to use have started integrating game mechanics and game design thinking - also known as gamification. Although, gamification has been used in several types of mobile applications (e.g., Waze [6] and Mobee [7]), the focus of this paper is on mobile applications that offer location and cultural information services to users. Adding gameplay to these applications opens up new opportunities to transfer education and culture in a more effective and efficient manner than the traditional learning methods (e.g., an audio guide). For example, the Ghost Game [8] is a mobile cultural heritage application that uses missions and quests in addition to photographs of actors with medieval dressing to connect visitors of the Wartburg castle in Germany with the past history. Gaming creates an immersive experience for visitors of the castle to connect with the people who lived in the medieval times. However, adding game design elements and mechanics alone in mobile guide applications does not guarantee that the intended learning occurs. This is because the dynamic nature of the player's situation in mobile environments rapidly affects their ability to process, store and respond to information. For example, the light and noise levels in outdoor environments may have a detrimental impact on the player's ability to complete a mission required to achieve a specific learning outcome. Therefore, there is a strong need for additional communication modalities in order to ensure player comprehension and understanding. The latest generation of mobile devices offer a plethora of sensors (e.g., ambient light sensors, accelerometers, gyroscopes, array of microphones, HD cameras) to enable multimodal communication with mobile applications. Embedding multimodal communication in gamified mobile guide applications can enable the creation of learning experiences close to game quality where players immerse deep in the educational scenario.

A standard feature used by games to immerse players in a scenario is artificial Non-Playable Characters (NPCs) who have a central role in the game's storyline. Those NPCs can utilize multiple communication modalities (body gestures and facial expressions, emotional recognition and reaction, etc.) and natural language to provide an embodied metaphor for implementing various gameplay mechanics (e.g., increasing task challenge through dialogue, narrate a story that helps gameplay, give the player a goal, etc.). Therefore, augmenting gamified mobile guide applications with a similar to games embodied metaphor (named Embodied Conversational Agents (ECAs)) could potentially result in enhanced learning by immersing players deeper into the educational scenario. ECAs are already a reality for the latest generation of mobile devices (e.g., [9]) and more projects are on the way for more technological breakthroughs (e.g., [10][11]). However, insufficient attention has been paid to the empirical evaluation of such interfaces. The direct consequence is that there is limited evidence on the potential impacts of ECAs on the players of gamified mobile guides. Given this lack of knowledge, there is a potential risk associated with the introduction of ECAs in gamified mobile applications. If the ECA does not actually enhance the application or it is not appropriate for the particular situation, the player may perform poorly, become distracted and the entire interaction may collapse.

The research framework presented in this paper provides researchers and practitioners with a common pillar to accumulate compare and integrate results from different studies. Although the framework focuses on the domain of gamified mobile guide applications, it can be useful to researchers in other domains where player learning is important and gamification using ECAs can be applied (e.g., mobile apps to support the development of literacy and maths skills). 


\section{The Underlying Theory}

\subsection{The CASA Paradigm}

The main reason to use ECAs in gamified mobile applications is to provide an interaction metaphor that mimics social face-to-face interactions between humans. In using this visual dimension of interaction, "natural" gameplay can be realised. In this type of gameplay, players interact with gamified applications without having to learn any complicated game commands, but rather in a natural and intuitive way [12], much like in a real-world game. However, it is still an open question whether the metaphor of human-to-human social behaviour can be freely applied to human-computer interaction. Some encouraging evidence can be found in the work of Reeves and Nass; "Computers-are-Social-Actors" (CASA) [13]. In a series of studies, the researchers showed that people respond to computers as a whole and ECAs ([14] [15]) specifically, in a social manner, much as they do with other people. For example, in one of their studies, they found that users perceived being monitored by an ECA as having the same effects, as those when being monitored by a real-human being. More recent studies (e.g., [16]) provided additional evidence that further reinforced the social aspect of ECAs. Although, this is encouraging news towards the general adoption of ECAs, it tells us nothing about whether the use of ECAs can actually provide a better paradigm for gamification than the existing methods (e.g., rich graphic elements [17]) alone. Then, as the CASA-related studies were done in stationary computer environments, mobile players may not experience the same social responses towards an ECA. This is because in stationary environments, players are isolated from the rest of the world, while in mobile environments they are constantly interacting with other people and their surroundings. However, for the purposes of this paper, we will not rule out the possibility that the CASA-findings may also apply to mobile gamified mobile guide applications using ECAs.

\subsection{The Theory of Multimedia Learning}

Another part of the theory behind ECAs is their effects on multimedia information processing. A popular model of how humans process multimedia information is the cognitive theory of multimedia learning [18]. The theory is based on three main assumptions: (a) humans process information through two separate channels (auditory and visual), (b) the channels have a limited capacity and (c) that learning occurs when the learner actively engages with the cognitive processes of filtering, selecting, organising and integrating with existing knowledge. In relation to ECAs, empirical evidence suggests [19] that the use of auditory input encoded in a personal conversational style results in deeper learning of the provided information in e-learning applications. Furthermore, the notion of redundancy shows that the visual embodiment of ECAs has an important role to play in augmenting the facilitation of information comprehension. Redundancy can be defined as the quantity of identical information provided simultaneously from different communication modalities (e.g., text and images). As redundancy increases, so is the likelihood that the information provided to be understood. This is because even if the information provided by one modality results in confusion and misunderstanding the modality can be supplemented by providing the same information through another modality at the same time (or approximately at the same time). Therefore, augmenting an ECA's verbal channel with additional modalities for information transfer (e.g., body gestures and facial expressions) should directly affect comprehension of the provided information. Previous research has investigated this hypothesis and has shown that ECAs capable of augmenting spoken dialogue with relevant nonverbal behaviours can increase the learning effectiveness of pedagogical applications (e.g., [20][21]). A recent empirical study [22] has also shown that augmenting an ECA-based pedagogical application with game elements (e.g., stars as form of reward) results in increased usability and player acceptance, as opposed to an ECA-based application without game elements. Although, additional research is needed to evaluate the impact of the fusion of game elements and ECAs on the player's comprehension of the subject, the existing findings reveal their strong potential in provoking enhanced player learning in pedagogical applications.

\subsection{The Simplex II Model}

Investigating the potential effects of gamifying mobile applications with ECAs on the player's comprehension of cultural narratives requires a more analytical architecture of human cognition, 
than the model of multimedia learning can provide. We have chosen the Simplex 2 (see Figure 1) model of human cognition [23], for two reasons: First, its application in investigating HCI problems has been validated by two large sample qualitative meta-analyses [24]. Similar theories of human intelligence (e.g., Sternberg's triarchic theory of human intelligence [25] have not yet been applied in this way. Second, the model captures a comprehensive understanding of human cognition, but it is yet simple enough to be easily adapted to assist in the design and evaluation of gamified mobile guides using ECAs. For example, the integration of Octalysis [26] with Simplex 2 was done with relative ease, as its architecture features a module for emotions and motivation. The model postulates nine modules of intelligent human behaviour, each of which can act partially independently of each other. Each module has implications for the design of multimodal instruction and the quality of the player's experience. In order to aid the understanding of the Simplex 2 model, we present each of these modules below, along with a discussion on the implications of each module for evaluating the cognitive accessibility of a gamified guide application design using ECAs [27]:

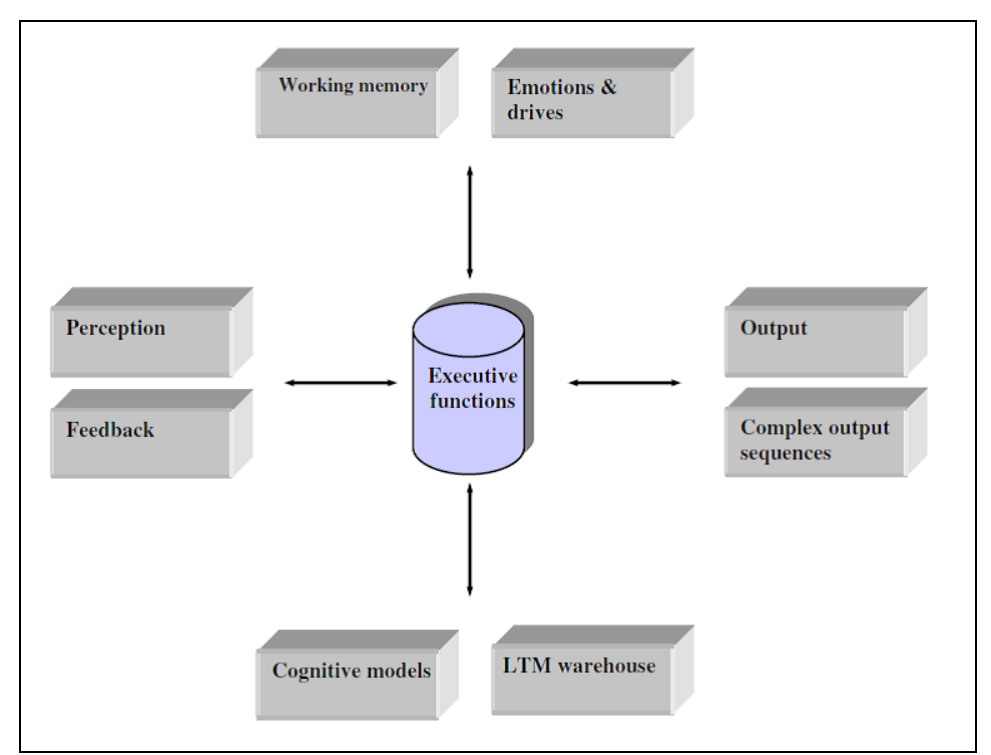

Figure 1. A depiction of Simplex II

\section{\# One: Executive Functions Module}

This module supervises, coordinates and controls the cognitive processes taking place in the other modules. It acts as a control node to the execution of cognitive processes in the model, transferring and transforming information between the relevant modules according to the demands of the cognitive task and the player's prior experience. The design of a gamified guide application is not accessible enough, when it puts excessive demands to players in terms of task complexity, its learnability and the organizational skills required to complete it successfully. Also, a design is not accessible enough when it is not consistent with the task at hand. An example when this module is overloaded is when players are required to complete tasks as part of a gamified tour guide experience that include many and hard-to-navigate routes. This task requires the intense coordination and control of several of the modules (e.g., working memory and cognitive models) to complete successfully.

\section{\# Two: Perception/Input Module}

This module takes sensory and perceptual information from the environment in the model. It holds, evaluates, maintains and rehearses information. The design of a gamified mobile guide application is not accessible enough when its input modalities (e.g., visual, auditory) are not adequate enough to match the sensory capabilities and skills of its intended players. An example when this occurs is when the design cannot automatically adjust to the light intensity around the mobile device to optimize the display viewability (e.g. to make the screen easier to see under bright sunlight conditions). 


\section{\# Three: Feedback Management Module}

This module deals with the feedback a player needs when working with a system. A gamified mobile guide application is not accessible enough when it provides inadequate feedback in terms of sensory (too brief or too loud), timing, relevance of information and memory requirements. A good example is when the output of the guide application is poorly synchronised (e.g., the ECA's speech and gestures with GUI game elements) or the ECA's speech quality is too low (e.g., too loud, too soft or gaps while speaking) disrupting the flow of information, which in turn causes confusion to players.

\section{\# Four: Working Memory Module}

This module operates as a working memory, providing, evaluating and transferring the required information to support the implementation of a cognitive task. Its storage capacity is limited. The design of a gamified mobile guide application is not accessible enough when the flow of cultural content is too dense or when the application cannot respond to potential cognitive overloads of the player's working memory (e.g., by automatically pause the flow of the cultural content).

\section{\# Five: Emotions and Drives Module}

This module generates the emotional responses that emerge from the player's motive or drive itself towards using a gamified application, from the cognitive events and actions caused by the motive and from the achievement or failure of the desired goal. A design that is not accessible enough, will receive inappropriate emotional responses, which in turn may discourage players from using the gamified application again. For example, the use of game elements and/or an ECA in a mobile guide application system may prove to be a frustrating or annoying experience or not be serious enough for the task at hand. To account for what motivates players we have extended this module to include the eight core drives of the Octalysis gamification framework (see Figure 2). The 8 core drives of gamification are: (1) Epic Meaning and Calling which is the need to participate in something bigger than just yourself. (2) The Development and accomplishment drive motivates people towards a specific goal and accomplishing it. (3) Empowerment of Creativity and Feedback is the core drive that motivates people to incorporate their creative into their daily lives. (4) The Ownership and Possession is the primary core drive that motivates people to accumulate possessions. (5) Social Influence and relatedness refer to the activities motivated by the influence of other people (e.g., by what other people do or think) (6) Scarcity and Impatience is what motivates people to want something they cannot have (e.g., because it is not immediately or easily obtainable). (7) Unpredictability and curiosity is the main force behind the peoples' obsession with experiences with unknown outcome and involving chance. (8) The Loss and Avoidance refers to the motivating factors that help people avoid situations they do not want happening (e.g., to die in a game).

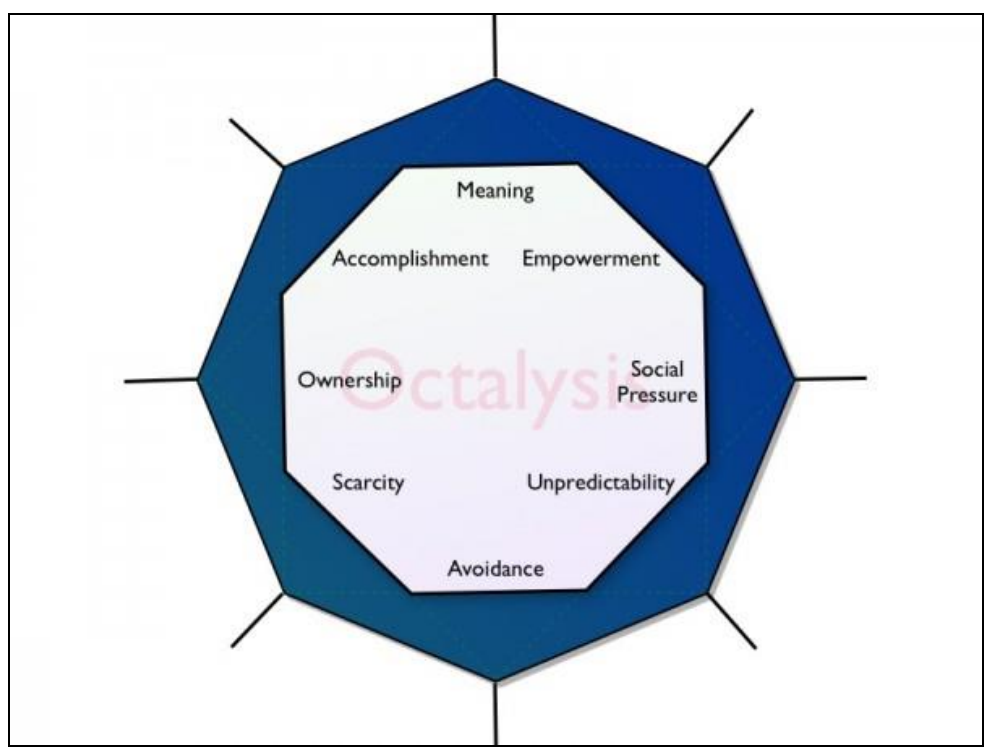

Figure 2. The Octalysis Gamification Framework (source: [26]) 


\section{\# Six: Long Term Memory Module}

The warehouse of long-term associative memory holds the knowledge and expertise that need to be retained on a long-term basis, including declarative and procedural knowledge. The design of a gamified mobile guide application is not accessible enough when it requires from players to rely too much on their long-term memory to form a coherent story about the cultural narrative or it does not provide sufficient support for long-term learning of routes and narratives or if the narratives are not well related to what the player already knows.

\section{\# Seven: Cognitive Model Construction Module}

This module acts as a facility for building, retaining and applying mental models or maps to solve practical problems. The design of a gamified mobile guide application is not accessible enough when the structure of the cultural narrative is difficult to follow and/or players have a hard time creating a mental map of the route they have to navigate or the information is not presented in an effective and accessible manner by the output modalities of the application. For example, certain hand gestures have different meaning in different countries. Their wrong use by an ECA can disrupt the conveyed information causing confusion to the player.

\section{\# Eight: Output Module}

This module retains and generates responses, as a consequence of the cognitive processes taken place in other modules and habitual responses, based upon triggers in the environment. The design of a gamified mobile guide application is not accessible enough when given the skills of its intended players, it requires from them to respond unreasonably to its requests or it does not provide sufficient support to successfully complete its requests. For example, the application may require from players to navigate routes that require complex decisions without providing sufficient support or motivation (e.g., to award points for correct navigation decisions)

\section{\# Nine: Complex Output Sequences Module}

This module augments the other output module, by supporting the development of complex responses. The design of a gamified mobile guide application is not accessible enough when given the skills of its intended players, it requires from them to uncover cultural narratives from locations that can reach after navigating complex routes or it does not provide sufficient support or motivation for players to successfully complete its requests.

The term cognitive accessibility ( $\operatorname{Cog} A)$ used above refers to the degree to which a player can cognitively access, interpret and respond to the information conveyed by an ECA (through speech and body gestures) and the application's game elements (e.g., points, step-by-step tutorials). Then, usability refers to the extent to which a player can interact with the ECA and the game elements to complete tasks with completeness (e.g., whether the player did a better job completing the tasks with the gamified mobile guide application), little effort (e.g., how quickly was the player able to complete the assigned tasks) and satisfaction [28]. From this discussion, it is clear that cognitive accessibility $(\operatorname{Cog} A)$ is a pre-condition for usability, but not vice-versa. If the design of the ECA and/or the game elements hamper the player's ability to cognitively access the cultural narratives of a mobile guide system, it is unlikely that s/he will be able to actually use the system to achieve the desired tasks. From this point of view, one could also argue that usability is a subset of cognitive accessibility, as even routine tasks require some degree of cognitive processing to complete successfully. Despite this strong relationship though, each term refers to different aspects of the interaction process and hence, should be kept and evaluated separately.

\subsection{The Theory of Distributed Cognition}

A theoretical framework for research in mobile applications, cannot be completed without considering cognition beyond the individual (as illustrated in Simplex 2) and to the environment as a whole. In such a context, there is the need to extend what is considered cognitive to encompass interactions between people and resources and materials in the environment. From the existing HCI theories, we have chosen distributed cognition (DCog) [29] as a suitable theory to understand these interactions. As opposed to similar theories (e.g., Activity theory [30]), DCog has three basic principles: (1) embodied cognition, (2) socially distributed cognition, and (3) culture \& cognition that are easy to understand, and can be adapted with minimal effort to ECAs and gamified mobile 
guide applications. The principles are elaborated below, supported by evidence from the literature about the potential impact of ECAs and gamification:

- The first principle of DCog holds that cognition is embodied, which is the idea that the development of cognitive processes in people is strongly influenced by their immediate environments. In other words, this approach postulates a strong and complex relationship between internal and external processes which involves coordination at many different time scales, between internal resources such as memory and attention, and external resources such as the objects and artefacts, constantly surrounding us. A critical moment in this coordination, that can decide its success, is whenever the player needs to switch their attention focus from the virtual world of the mobile device, to the actual objects/artefacts of the physical world. An ECA could be used to effectively guide the player's attention focus towards these physical objects by helping the player in understanding the underlying structure of the physical space. It may, for example, help the player to locate a needed landmark when navigating a complex route by directing their gaze (through the use of proper gestures) towards its current location in the virtual world. This should allow the player to better locate the object in the physical environment.

- The second principle of distributed cognition dictates that cognitive processes are distributed across the members of a group, according to the group's social organization. It can be argued that this form of cognition is driven by social elements such as, mentorship, acceptance, companionship, competition and envy. People's interactions in a group are influenced by and relate to other people. When the patterns of these interactions are stable, they reflect some form of underlying cognitive process. For example, a group member that excels in an area will motivate other group members to reach the same level. Then, group members are inclined to draw closer to other group members they can relate to (e.g., from the same hometown or with common interests). There is evidence in the literature that ECAs can improve the level of collaboration of students using interactive games [31], but have no impact on the realization of short-term learning objectives.

- The third principle of distributed cognition holds that cognition is not isolated or separable from culture, because people live in complex cultural environments. In this perspective, culture shapes the cognitive processes of people, artefacts and environments. The significance of gamification in culture and society as a whole has only recently started to be recognised. In education, a number of advocates of game-based learning see the development of educational games as a moral imperative, as learners of the "video game generation" do not respond well to traditional instruction [32][33]. Then, in a wider recognition of the social and cultural importance of gamification, Raessens [34] sees a general "ludic turn" of the society where games take their place alongside film, radio and television in their impact on the production and reproduction on identity and culture. In relation to ECAs, the literature suggests [14][15] that humans seek the same cultural traits in their communication with ECAs as they would in human-human communication. For example, Cowell \& Stanney [35] showed that when players are given a choice to select a virtual character from a pool of characters with different ethnicities to work within a cooperative task, they would select a character that matches their own ethnicity.

Having included distributed cognition (DCog) in our theoretical framework, the terms of cognitive accessibility and usability are no longer sufficient to cover players and their interactions in whole environments. A more holistic, all-encompassing notion is needed to describe effectively those settings. The player experience, that is the overall experience players get when they interact with an ECA in a gamified application, fully satisfies this description. More specifically, the player experience refers to the cognitive accessibility and usability of an ECA-based gamified interface, and includes a number of factors that influence the experience the interaction evokes. Below, we identify these factors as part of the player's journey. Our research framework comes next that includes a number of suggested variables that could be empirically investigated.

\section{The Player's Journey}

The player's journey through a gamified application can be broken down into 4 distinct phases that needs to be optimised for player experience: (1) Discovery, (2) Onboarding, (3) Scaffolding, and 
the (4) Endgame [36] (see Figure 3). Using the theoretical framework as a foundation, we can identify the mechanisms of cognition and the factors that influence the player's experience in each phase of the journey. As discussed above, the mechanisms of cognition are either related to the individual and/or are distributed among people and the environment. The factors affecting the player's experience can be intrinsic, extrinsic or both. To further investigate these factors we have identified a number of appropriate variables (see our proposed research framework) to evaluate existing design problems in gamified mobile guide applications using ECAs and generate new technological developments and new design solutions. The player's journey starts at the discovery stage, where the player first discovers and learns about the gamified application. The hard part of this phase is to mentally prepare the player to start and engage with the journey. To ensure a positive player attitude during this initial awareness stage, a design needs to be optimized for: (1) Perception and Input, (2) Emotions and Drives, (3) Executive Functions, and (4) Socially and Cultural Distributed Cognition. A factor that may impact the output of these cognitive mechanisms and hence, how accessible and/or usable a player finds a gamified application with an ECA is the intrinsic qualities of the player (see \#Factor 1: Qualities of the Player). This phase ends when the player has started using the product. In the next phase (Onboarding phase), the player needs to become familiar with the rules and the tools (e.g., game mechanics, win-states) to play the gamified application. The emphasis in this stage should be on why players need to use the application and less on how they should use the application. To ensure that players have mastered the fundamental skills needed to use the application and to achieve the early-stage win states a design needs to be optimised for the cognitive mechanisms of the previous phase, as well as for: (1) Working Memory, (2) Feedback Management and (3) Cognitive Model Construction. The two extrinsic factors that may impact the output of these mechanisms is the attributes of the gamified task (\#Factor 2: Features of the gamified task) and the attributes of the ECA (\#Factor 3: Features of the ECA). This phase ends when the players are fully equipped and ready to embark on the journey on their own. The third stage in the player's journey is Scaffolding. This is when players use what they learnt during the onboarding phase to try to achieve as many win-states as possible. To ensure that players will come back on a regular or daily basis to commit to the educational activities of the gamified mobile application a design needs to be optimised for the cognitive mechanisms of the onboarding phase as well as for: (1) Output; (2) Long Term Memory; and (3) Embodied Cognition. This phase ends when the players believe they have explored and tried what the application has to offer at least one time. The fourth and final phase in the player's journey is the Endgame. This is when the player starts feeling there are no longer unexplored features of the application. The hard part of this phase is to keep the player interested, so it never becomes boring to them. In this phase, a design should enable veteran players to develop complex output responses by utilizing what they already know about the gamified application in new and innovative ways. An extrinsic factor that can influence the output of the cognitive mechanisms in both phases is the attributes of the mobile environment players interact with (\#Factor 4: Mobile Guide Enviroment).

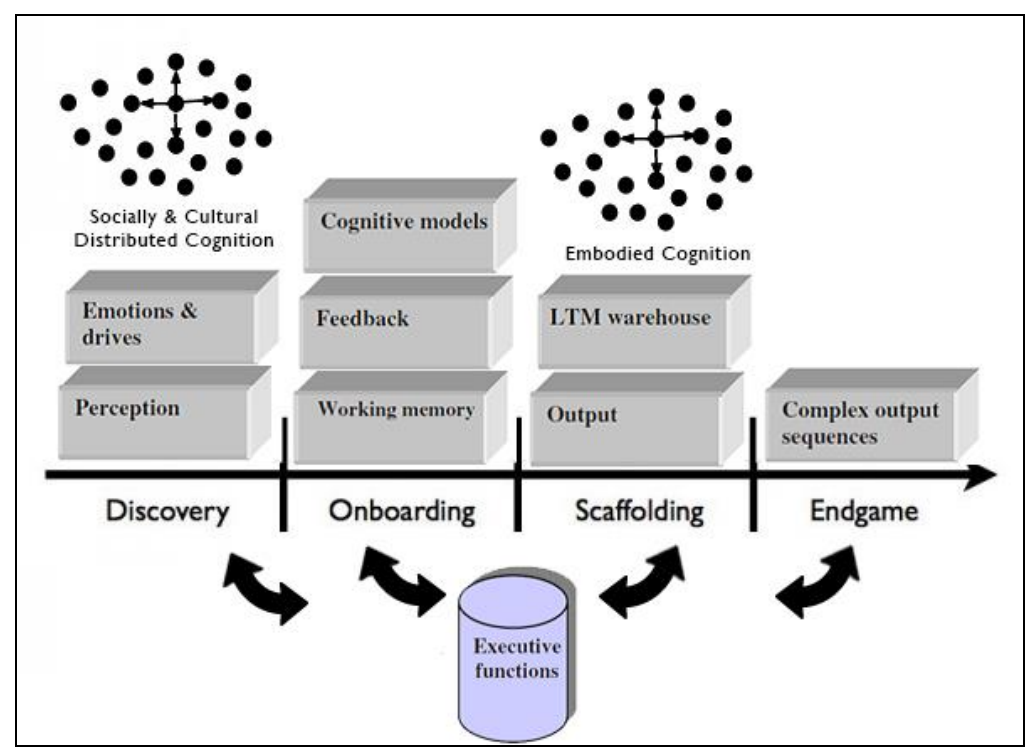

Figure 3. A depiction of the player's journey showing the new cognitive mechanisms introduced in each phase 


\section{A Framework for Research in Gamified Mobile Guide Applications using ECAs}

Our proposed research framework, draws on the theories discussed above and previous work in establishing a research framework for ECAs [37]. For each of the factors identified in the players' journey we propose a number of variables that we think an empirical study should start from. The list is by no means definite or conclusive as more variables are possible within each factor. We conclude our discussion with the variables that we have tested in our empirical studies.

\section{\# Factor 1: Qualities of the Player}

Potential players vary, of course, in many ways. However, based on the cognitive mechanisms we identified in the discovery phase of the player's journey, we can derive certain qualities, that may quite likely to affect the player's experience. These qualities include:

Player Gender: The player's gender can play an important role in the player's overall perception and task performance with gamified mobile applications using ECAs. Recent research in the general gaming preferences and behaviours of current video game players [38] reveals that female players are more likely than males to play games from the gamified and Educational/Edutainment genres. In our empirical works, we found evidence that the attention grabbing strategies used by an ECA [48] impacted the retention of cultural narratives differently between participants of different gender.

Player Age: The player's perceptual capability can be differentiated greatly among players of different age groups. Players of older age groups often have reduced perceptual and cognitive abilities as well as motor responses compared to those in the younger age groups. Therefore, it is reasonable to expect that the player's attitudes and task performance when interacting with a gamified application using an ECA also vary across different age groups.

Player Type: Bartle [39] categorised players in four categories according to the player's personality traits: 1) Achievers: These are players who play to gain "points", equipment, rewards, progress levels, and other concrete measurements of succeeding in a game. 2) Explorers play to discover the area, and hidden places. They will go at great lengths to discover an unknown glitch or an easter egg. 3) Socialisers will choose to play a game for the social aspect rather than the actual game itself. 4) The killer players thrive on competition with other players and prefer fighting NPCs (Non-Playable Characters) as a way to progress in a game. The GamerDNA test can categorise players according to the type they mostly are, as it is unlikely a player to be of only one type. Different player types have different motivations, perceptions and may perform differently in a gamified mobile task featuring an ECA. For example, a player who is mainly of a killer type may find a gamified task without the element of competition as tiresome and dull.

Player Ethnicity: We have found evidence that the player's race impacts the way a player perceives an ECA. In particular, in our empirical studies in Greece, we found evidence that players would like an ECA that has more Greek-like characteristics [1]. However, it is still an open question how participants perceive a gamified task where the misinterpretations of their race (e.g., Blacks, Greeks, or other races) found in other media (e.g., Television) are present in the game. According to a research done in [40] most of the players who use games where racism and misinterpretation of blacks and Latinos are prevalent (e.g., Grand Theft Auto), are themselves part of those minorities or races. This shows that for players of video games a game is "just a game". However, this argument should not be applied in gamified mobile applications using ECAs. Due to the scarcity of previous research in the area, it is an open question how the players' race can influence the perception of those applications under realistic mobile conditions.

Player Class: The cultural homology perspective [41] [42] asserts that individuals in upper classes consume distinctively different types of cultural products than individuals in middle or working class occupations. A Google search suggests that this perspective applies to the consumption of video games as well. Specifically, members of the upper class are the main consumers of highcultural products among which is art-games [43]. Such games are a form of gamified applications designed to emphasise art or whose premise is intended to produce some kind of non-ludological reaction to its audience. On the other hand, middle and working classes seem to enjoy more mass 
culture and some surprisingly even violent crime video games (e.g., Grand Theft Auto) [44]. Therefore, it is reasonable to hypothesise that gamified mobile guide applications using ECAs will be more appealing to upper class players. However, to the best of our knowledge, there has not been so far an empirical study where the impact of the player's class has been investigated on their satisfaction and task performance with gamified mobile applications (of any kind). Hence, this remains an open question.

Other variables: Other player-related variables are "background knowledge" and "device experience"

\section{\# Factor 2: Features of the gamified task}

The gamified task in which the player is asked to perform with the aid of the ECA can also vary in many different ways. Some of these features are:

Gamification elements: There are numerous gamification design elements that can be used to make an educational task as fun and engaging as a game. Each of these elements can be used in a form of ECA-driven scenarios and/or standard GUI elements. The Octalysis framework includes a number of game design elements that can be used as appropriate in the optimization of the experience in each phase of the player's journey. These elements include: PBLs (points, badges, leaderboards), narratives, appointment dynamics, thank-you economy etc. We have selected the elements that we think are relevant to the context of mobile guide applications and mapped them to the four phases of the player's journey (see Table 1). A detailed explanation of the elements can be found in [45] [46]. The list is not meant to be conclusive as additional elements could be added in each phase and/or some elements may not be appropriate. In our prototype mobile guide systems we used narratives in all phases of the player's journey, step-by-step tutorials in the onboarding phase and milestone unlocks in the scaffolding phase.

Table 1. Gamification Design Elements mapped against the phases of the player's journey

\begin{tabular}{|c|c|}
\hline Gamification Design Elements & Player's Journey Phases \\
\hline $\begin{array}{l}\text { - } \text { Narrative (or story) } \\
\text { - Elitism } \\
\text { - } \text { FOMO (Fear of Missing Out) } \\
\text { - } \text { Grial Treasure/Gifting } \\
\text { - Scarlet letter (fear of becoming the "loser") } \\
\text { - Evanescence }\end{array}$ & Discovery \\
\hline $\begin{array}{ll}\text { - } & \text { Step-by-step tutorial } \\
\text { - } & \text { Glowing Choice } \\
\text { - } & \text { First boss fight (first major win-state) } \\
\text { - } & \text { Narrative (or story) } \\
\text { - } & \text { Points } \\
\text { - } & \text { Badges } \\
\text { - } & \text { Leaderboards } \\
\text { - } & \text { Progress Bar } \\
\end{array}$ & Onboarding \\
\hline $\begin{array}{ll}\text { - } & \text { Milestone unlocks } \\
\text { - } & \text { Narrative (or story) } \\
\text { - } & \text { Meaningful Choices } \\
\text { - } & \text { Social Prodding } \\
\text { - } & \text { Thank you Economy } \\
\text { - } & \text { Easter Eggs } \\
\text { - } & \text { Suspense } \\
\text { - } & \text { Evanescence Opportunity }\end{array}$ & Scaffolding \\
\hline $\begin{array}{ll}\text { - } & \text { Elitism } \\
\text { - } & \text { Narrative (or story) } \\
\text { - } & \text { Rolling Rewards } \\
\text { - } & \text { Social Treasure/Gifting } \\
\text { - } & \text { Collection Sets } \\
\text { - } & \text { Evergreen Mechanics }\end{array}$ & Endgame \\
\hline
\end{tabular}

Personalisation of narratives: An important feature of mobile tour guide applications is the ability to provide narratives, tailored to the current physical location and the player's profile. Of 
course, different types of personalised narratives may have a different impact on the player's attitudes and the ability to recall information from cultural narratives. Although, more research is needed to define the appropriate types of personalisation and values for each type, a systematic exploration could be started by considering the types narrative difficulty with values simple and technical narratives and personal interests with values Architecture, History and Biographical related information.

Navigation difficulty: The difficulty of a navigation task might be simple, in which players have to achieve a series of milestones consisted of finding landmarks and locations that are easy to locate to successfully complete the task. Alternatively, a player may carry out a more difficult navigation task where they have to achieve more complex milestones (consisted of harder to find landmarks and locations) to successfully complete the task. An ECA could impact differently how difficult players find each type of route and on the efficacy of the navigation task.

Competition: How players perceive and perform in tasks with gamified mobile guide applications is most likely affected by whether and how the task introduces competition. A competitive task may require players to compete against time, against other players or against themselves. From these options, we view a treasure-hunt style of guidance as an effective way of introducing competitive tasks for players who want to experience them (e.g., long-term visitors of an attraction). Such tasks require players to achieve a goal (e.g., to find an item) by uncovering clues hidden in the system's narratives about a specific tour. Players who choose this method to experience cultural narratives should be able to view the task performance of other players and who was able to achieve the final goal. It is an open question how competitive tasks (as opposed to non-competitive tasks) affects the perception and task performance of players experiencing cultural narratives with ECAs in mobile environments.

\section{\# Factor 3: Features of the ECA}

Like players, ECAs can vary in a wide variety across several features. These features include:

Visual presence: There is a limited empirical evidence to support the argument that conversational agents should be embodied in mobile guide interfaces. Additional research is needed to establish the impact of ECAs in mobile guide interfaces. For example, it could be found that voice or text alone is sufficient for the interaction to take place and for tasks to be completed successfully.

Multimodal behaviours: An important component of the ECA's multimodal abilities is that of sensing the player and react with appropriate behaviours. Although computer vision technologies have endowed ECAs with the ability to sense various features of the player (e.g., emotions, head position, etc.), it is how an ECA reacts to these multimodal inputs that can impact the player's satisfaction and task performance. For example, an ECA can use humour or sarcasm as an effective strategy to get the player's attention back to the task at hand, when it was lost. On the contrary, a too forceful strategy will probably irritate players and disrupt them from the successful completion of the task.

Domain experience: In the context of gamified mobile guide applications we hypothesised (see study five), that an ECA that uses minimal non-verbal behaviours would most likely be perceived as a less experienced tour guide than an ECA that uses a range of non-verbal behaviours. This hypothesis is according to the real-life life scenario, where visitors of a tourist attraction will most likely find a human guide who makes sensible use of both verbal and non-verbal channels as more experienced than a human guide who speaks continuously and displays minimal non-verbal cues.

Natural language capabilities: The ability of an ECA to process natural language and respond appropriately is important for a wide range of player-ECA dialogue tasks (e.g., Q\&A dialogues, setting goals to achieve (e.g., find a location), providing feedback, reward achievement, etc.). Script-based approaches are generally very robust in matching an input question to an answer in the database. However, scripts require large databases with all possible Q\&A pairs for a single question to work effectively. An alternative is to process the semantics of human language, which, however, requires the input to be formed in the correct grammatical form. This is most likely to result in players having to rephrase the same question several times. Although this could benefit 
task performance (as the player has to review the content a number of times to rephrase the question), it may have a detrimental impact to the overall player's experience.

Other variables: Other ECA-related variables are: "amount of embodiment", "modality of communication", "type of non-verbal cues", and "ECA gender".

\section{\# Factor 4: Mobile Guide Environment}

The environment in which the mobile guide operates can, of course, varies in many ways. These include:

Indoor/Outdoor: We distinguish two types of mobile environments for which a mobile guide system and an ECA should be tailored for: an indoor mobile environment (e.g., a museum) and an outdoor mobile environment (e.g., a castle). Because of the unique nature of each environment, the potential impact of ECAs on the player's experience is also distinctive. For example, in an outdoor environment the task of uncovering information about an attraction may be, by itself, a less efficient task than in an indoor environment. This is because the player may have to devote more mental and physical resources to complete the assigned task. For this reason, it cannot be assumed that an ECA has the same impact on the player's experience across the two environment types.

Other variables: Other environment-related variables are: "features of the environment".

In the studies reported below, we addressed the following eight variables: player gender, ECA visual presence, ECA domain experience, natural language abilities, multimodal behaviours, navigation difficulty, and personalisation of narratives.

\section{Experimental Studies}

A series of six experimental studies were conducted within the above research framework, with the aim to investigate the impact of the above variables on the player experience of mobile guide applications. The studies were conducted in the laboratory under simulated conditions using highfidelity mobile guide prototypes. To simulate the routes and attractions, participants would visit in a real medieval castle (namely the castle of Monemvasia), we used immersive panoramic and video interactive applications projected on the wall of the lab. The mobile guide applications included a realistic female ECA capable of a range of verbal and non-verbal behaviours in the role of a tour guide of the castle. In total, 97 participants took place in all six studies from a variety of academic and mobile computing backgrounds. A variety of methods were used for data collection including, post-task interviews, 7-point Likert questionnaires [47], written retention tests, protocol analysis, eye-tracking and facial expression. Table 2 shows a synoptic view of the design of the six experimental studies. This is followed by a brief description of each study and their major findings. An analytical discussion of the studies and their results can be found in [48] [49] [1] [50] [51].

Table 2. A synoptic overview of the design of the six experimental studies

\begin{tabular}{|c|c|c|c|c|}
\hline Studies & $\begin{array}{l}\text { Laboratory } \\
\text { Setting }\end{array}$ & Users & Research Goals & Conditions \\
\hline Study 1 & $\begin{array}{l}\text { Immersive } 360^{\circ} \\
\text { panoramic } \\
\text { display }\end{array}$ & 21 & $\begin{array}{l}\text { Investigate the impact of the presence of a } \\
\text { multimodal ECA on the retention of cultural } \\
\text { narratives the efficacy of the navigation task }\end{array}$ & $\begin{array}{l}\text { Multimodal ECA } \\
\text { vs. Non-ECA } \\
\text { control }\end{array}$ \\
\hline Study 2 & $\begin{array}{l}\text { Immersive } 360^{\circ} \\
\text { panoramic } \\
\text { display }\end{array}$ & 17 & $\begin{array}{l}\text { Investigate the impact of the presence of an } \\
\text { ECA on the retention of cultural narratives of } \\
\text { variable difficulty }\end{array}$ & $\begin{array}{l}\text { ECA vs. Non-ECA } \\
\text { control and Simple } \\
\text { vs. Technical }\end{array}$ \\
\hline Study 3 & $\begin{array}{l}\text { Immersive video } \\
\text { application } \\
\text { display }\end{array}$ & 19 & $\begin{array}{l}\text { Investigate the impact of the presence of an } \\
\text { ECA on the efficacy of navigation with routes } \\
\text { of variable difficulty }\end{array}$ & $\begin{array}{l}\text { ECA vs. Non-ECA } \\
\text { control and Simple } \\
\text { vs. Complex }\end{array}$ \\
\hline Study 4 & $\begin{array}{l}\text { Immersive } 360^{\circ} \\
\text { panoramic } \\
\text { display }\end{array}$ & 15 & $\begin{array}{l}\text { Investigate how different techniques for } \\
\text { dialogue processing and the style of dialogue } \\
\text { can impact the retention of cultural narratives }\end{array}$ & $\begin{array}{l}\text { Script-based vs. } \\
\text { Parsing-based }\end{array}$ \\
\hline Study 5 & $\begin{array}{l}\text { Immersive } 360^{\circ} \\
\text { panoramic }\end{array}$ & 12 & $\begin{array}{l}\text { Investigate the impact of the ECA domain } \\
\text { experience on the retention of cultural }\end{array}$ & $\begin{array}{l}\text { Fully experienced } \\
\text { ECA vs. Low }\end{array}$ \\
\hline
\end{tabular}




\begin{tabular}{l|l|l|l|l}
\hline & display & narratives & Experienced ECA \\
\hline Study 6 & $\begin{array}{l}\text { Immersive 360 } \\
\text { panoramic } \\
\text { display }\end{array}$ & 13 & $\begin{array}{l}\text { Investigate the impact of the ECA's } \\
\text { multimodal behaviours on the retention of } \\
\text { cultural narratives }\end{array}$ & $\begin{array}{l}\text { Attention grabbing } \\
\text { ECA vs. Non- } \\
\text { Attention grabbing } \\
\text { ECA }\end{array}$ \\
\hline
\end{tabular}

The first study [1], evaluated the impact of a multimodal ECA on the cognitive accessibility and usability of a prototype tour guide system. In this study, a prototype system with a multimodal ECA was compared with a non-ECA control (that uses voice and text-subtitles). The potential effects of the ECA on the retention of personalised cultural narratives (with type personal interest) and the efficacy of navigation on routes of the castle were both addressed. To successfully navigate a route players had to unlock a series of milestones consisted of discovering landmarks and locations of the castle. The findings of the study suggest that the presence of the multimodal ECA had a zero effect (neither detrimental or beneficial) on the participants' ability to recall cultural narratives personalised according to their personal interests and from different locations of the castle. However, there was a positive impact of the ECA on the participants' ability to navigate routes that required slightly more complex navigation decisions. One last important finding that emerged from the study is that the degree of narrative personalisation can affect the participants' attitudes towards the mobile guide systems, the gamified tasks and the ECA Therefore, the primary focus of a system's designer should be on improving the cognitive accessibility of the cultural content, with the improvement of the system's technical performance to be a secondary goal.

The ECA zero effect was repeated in the second study [50]. In this study, we varied the narration between the ECA and the non-ECA control more aggressively by exposing participants to simple and technical narratives. We found that the visual presence of the ECA had neither a detrimental or beneficial impact on the participants' ability to recall information from narrations with different degree of difficulty. However, we also found that a visual ECA with text as an additional output modality can positively impact the participants' attitudes towards the technical content which could potentially lead to enhanced retention of technical information.

In the third study [51][1], we varied the navigation difficulty between the ECA and non-ECA control more aggressively by exposing participants to simple and complex to navigate routes. We found an ECA zero effect (neither beneficial nor detrimental) on the participants' ability to navigate routes with different degree of difficulty. However, participants thought that the navigation instructions presented by the ECA were clearer and better presented than the non-ECA control. Furthermore, participants thought that the more difficult to navigate route was more interesting when they experienced it with the ECA than with the non-ECA control. Therefore, it was concluded that although an ECA does not enhance the participant's ability to navigate routes of different difficulty, it was more useful in helping participants to decide where to go than the non-ECA control.

The fourth study [49] examined the variable natural language capabilities of ECAs. In particular, it investigated how the two popular approaches to processing natural language (a script-based [52] and a parser-based approach [1]) and specific styles of Q\&A can affect the participants' retention of cultural narratives and quality of the player's experience. We found that retention of cultural narratives is directly affected by the accuracy of the method for processing natural language in Q\&A. In the study scripts outperformed parsing in terms of the accuracy of answers provided to input questions. This gave participants less chances of becoming distracted and to overlook important pieces of information in the narratives. Then, we found that when the desired output of a Q\&A session is enhanced retention, participants should be motivated by the ECA to ask as many questions as possible about the narratives. This encourages participants to revisit the narratives more than once to come up with the required questions which in turn, could lead to enhanced retention. However, special care should be taken to properly motivate participants to avoid becoming frustrated by the repetitive activity. A last important finding is about when the Q\&A system fails to return an answer to the player's question. To ensure an optimal Q\&A session, the system should request players to repeat a question once. Alternatively, the system's fail-safe responses should be built in a way that enable players to figure out how to properly ask questions to avoid this type of responses.

In the fifth study [1] we investigated the impact of the ECA's domain experience on the retention of cultural narratives. It was assumed that domain experience in this scenario is the ability of the ECA to effectively use non-verbal means, and pauses in speech to augment verbal communication. We found evidence of the importance of the ECA's body language and pauses in speech in presenting cultural heritage narratives. In particular, the study showed that the mere presence of an 
ECA on a mobile interface is not enough to engage participants with cultural narratives. For participants to consider the ECA as part of the experience it needs to display relevant and wellsynchronised verbal and non-verbal behaviours.

The sixth and final study investigated the impact of an ECA's output multimodal behaviours on the retention of cultural narratives using a custom research method [48]. Participants experienced a mobile guide application featuring an ECA that portrayed two types of attention-grabbing multimodal behaviours (humorous and serious) and a control application with an ECA without any attention-grabbing multimodal behaviours. We found that an ECA should not attract attention to itself more than necessary to avoid becoming a distraction from the flow of the narration. We also found that the attention-grabbing ECA had an inverse impact on the retention of cultural narratives in participants with different genders. Specifically, the ECA affected the female participants more than the male participants. Therefore, even when an ECA can autonomously react to the player's attentional state, a minimum threshold where the ECA should request the participant's attention should be established. This threshold is difficult to determine as the ECA must avoid becoming tiresome, but also must be effective enough to attract the participants' attention when it has diviated. A last finding that emerged from the study was that the multiple communication channels used by ECAs can result into increased cognitive workload for elderly participants. Hence, for those participants, designers should give the option to use conventional channels of communication such as text and voice to experience cultural narratives in gamified mobile guide applications.

\section{Design Guidelines}

On the basis of the findings of the empirical studies reported above, we have established a number of guidelines for optimising the player experience (as defined in this paper) in gamified mobile guide applications using ECAs. The guidelines are based on evidence generated from the studies and the authors' experience gained from the design and development of a number of ECA-based gamified guide applications for mobile devices. In total we have identified six categories and thirty-four recommendations that are presented in layman terms below:

\subsection{ECA-Design}

\begin{tabular}{l|l}
\hline Sources & \multicolumn{1}{|c}{$\begin{array}{l}\text { Enable player-modified avatars. Allow players to modify the appearance of the ECA to } \\
\text { match their individual preferences and needs. For example, empirical studies with Greek } \\
\text { participants suggest that players would prefer an ECA with a more Greek-like appearance. } \\
\text { Prefer an ECA with non-verbal cues over an ECA without such cues. We found evidence } \\
\text { that body gestures and facial cues (e.g., smile, eye contact) make the player more visually } \\
\text { involved with the content than not having these cues at all. This could potentially lead to a } \\
\text { better retention of the narrated content. }\end{array}$} \\
\hline Study 5 & $\begin{array}{l}\text { Each ECA gesture should match accurately what is being said verbally. Participants in all } \\
\text { of the studies suggested that the body language of the ECA should be improved. An ECA that } \\
\text { displays asynchronously to the speech, gestures distracts players from the content it presents. }\end{array}$ \\
\hline All studies & $\begin{array}{l}\text { Ensure one gesture per sentence of the content. If you attempt to synchronise more than one } \\
\text { gesture per sentence of the content, the gestures will most likely overlap making the character } \\
\text { look unrealistic. }\end{array}$ \\
\hline expertience & $\begin{array}{l}\text { Avoid displaying negative facial expressions. Although participants in the studies did not } \\
\text { notice the ECA's negative facial expressions, it is best to avoid them as they make the character } \\
\text { look unrealistic. This is because the current ECA technology cannot offer consistent levels of } \\
\text { behavioural fidelity to avoid the uncanny valley effect [53]. Adding negative facial expressions } \\
\text { to an ECA, that looks like a human being, but does not exactly behave like one, will increase } \\
\text { this effect and make the character look repulsive and less natural. }\end{array}$ \\
\hline $\begin{array}{l}\text { Ensure optimal reactions to multimodal input. The ECA should respond to multimodal input } \\
\text { (e.g., to request the player's attention) with a maximum one second delay and without the } \\
\text { player's intervention. In any other case, enable players to turn-off the ECA's reactions and } \\
\text { move on with the narration. }\end{array}$ \\
\hline Study 1, \\
\hline Study 6
\end{tabular}




\subsection{ECA-Voice}

\begin{tabular}{|c|c|}
\hline Sources & \\
\hline $\begin{array}{l}\text { Expert } \\
\text { Knowledge }\end{array}$ & $\begin{array}{l}\text { Avoid using the same voice tone. Vary the way the ECA speaks to add realism to narratives. } \\
\text { For example, the ECA could talk faster, slower or stop speaking to attract attention. }\end{array}$ \\
\hline Study 5 & $\begin{array}{l}\text { Adopt a moderate rate of speaking. If you are using a Text-to-Speech (T2S) engine, make } \\
\text { sure you adjust its settings to create a moderate rate of speaking. If the voice still feels fast and } \\
\text { unrealistic, introduce a } 1 \mathrm{~s} \text { pause between the sentences of the content. }\end{array}$ \\
\hline All studies & $\begin{array}{l}\text { Prefer the voice of a real-human. The results from the studies suggest that participants prefer } \\
\text { the voice of a real human instead of a voice generated by a text-to-speech (T2S) engine. }\end{array}$ \\
\hline
\end{tabular}

\subsection{Multimodal Narrative/Content Design}

\begin{tabular}{|c|c|}
\hline Sources & \\
\hline $\begin{array}{l}\text { Expert } \\
\text { Experience }\end{array}$ & $\begin{array}{l}\text { Have an expert human author to create cultural heritage narratives. The content should be } \\
\text { created by a human author for everyday spoken use. }\end{array}$ \\
\hline$[1]$ & $\begin{array}{l}\text { Enable automated creation of multimodal narratives. Provide the necessary tools to enable } \\
\text { content authors to automatically add multimodal interactions (e.g., ECA body animations, face } \\
\text { expressions, voice intonation, etc.) to arbitrary narratives and other content. }\end{array}$ \\
\hline Study 1 & $\begin{array}{l}\text { Prefer historical narratives, when rapid prototyping of a guide system is needed. Study } 1 \\
\text { suggest that the majority of players prefer to explore historical over other types of narratives } \\
\text { when they visit an archaeological attraction. }\end{array}$ \\
\hline Study 1 & $\begin{array}{l}\text { Simplify technical narratives to just their plot. Narratives that require some technical } \\
\text { expertise (e.g., architectural information about buildings of the Monemvasia castle) could be } \\
\text { simplified to a series of causal events using minimal technical language. }\end{array}$ \\
\hline Study 1 & $\begin{array}{l}\text { Do not overwhelm players with historical dates. An ECA narrating some historical facts } \\
\text { shows credibility, but do not overwhelm players with too many historical dates. }\end{array}$ \\
\hline Study 1 & $\begin{array}{l}\text { Personalise the narrations when necessary. Include opinions and/or personal experiences to } \\
\text { make the narration more realistic. For example, "the construction of the sea wall of the castle } \\
\text { really catches your breath, don't you think?" }\end{array}$ \\
\hline $\begin{array}{l}\text { Study } 1 \\
\text { Study } 6\end{array}$ & $\begin{array}{l}\text { Author content that is "unexpected" and "spontaneous". Some examples are: a humorous } \\
\text { answer to a question posed by the player or an unexpected reaction to the player's lack of } \\
\text { attentiveness to a narration. }\end{array}$ \\
\hline $\begin{array}{l}\text { Study } 1 \\
\text { Study } 2 \\
\text { Study } 3\end{array}$ & $\begin{array}{l}\text { Author content that is concise and right to the point. Author narratives with short sentences } \\
\text { and without formalities (e.g., to be polite). Keep the navigation instructions to the absolute } \\
\text { minimum. }\end{array}$ \\
\hline Study 1 & $\begin{array}{l}\text { Create narratives that match the time-constraints of the visitors. The narratives should } \\
\text { match the time-constraints of the visitors. For example, short stay visitors will most likely } \\
\text { appreciate narrations of general interest about the castle. On the other hand, long stay visitors } \\
\text { will most likely want to experience in full what the castle has to offer and ask for more } \\
\text { elaborated narratives. }\end{array}$ \\
\hline Study 1 & $\begin{array}{l}\text { Author content of general interest. The results from the first study, suggest that participants } \\
\text { would like narratives of general interest (e.g., about local shops, dances, etc.) to be included as a } \\
\text { personalisation option in the prototypes. }\end{array}$ \\
\hline
\end{tabular}

\subsection{Mobile Guide Application Design}

\begin{tabular}{|c|c|}
\hline Sources & \\
\hline $\begin{array}{l}\text { Expert } \\
\text { Experience }\end{array}$ & $\begin{array}{l}\text { Avoid using menu-based dialogues in Q\&A with an ECA. Unless built with an authoring } \\
\text { tool, our experience suggests that menu-based dialogues are very time consuming to build and } \\
\text { debug and are not easily extendible. The use of natural language is highly recommended as an } \\
\text { alternative. }\end{array}$ \\
\hline All studies & $\begin{array}{l}\text { Design for touch interaction and for the latest generation of mobile hardware. Ensure the } \\
\text { player interface elements (e.g., buttons, dialogue menus, etc.) on the application are large } \\
\text { enough to avoid the "fat-finger" effect. Furthermore, avoid using hover effects (e.g., to } \\
\text { highlight interface items) and right click menus. Finally, design the system's layout for both } \\
\text { portrait and landscape orientations. }\end{array}$ \\
\hline Study 2 & $\begin{array}{l}\text { Use text as an additional output modality when presenting technical narrations. We found } \\
\text { evidence in study } 2 \text {, that the careful use of text as an additional output modality of ECAs when } \\
\text { narrating technical content can be beneficial for players. We recommend the style of text used } \\
\text { in the study, that is, auto-scrolling subtitles synchronised with the narratives. }\end{array}$ \\
\hline
\end{tabular}




\begin{tabular}{l|l}
\hline Study 3 & $\begin{array}{l}\text { Avoid using text in navigation instructions. We found no evidence that using text when } \\
\text { giving navigation instructions is beneficial for players. On the contrary, an ECA with a relevant } \\
\text { body language was perceived as more useful in helping participants taking the correct } \\
\text { navigation decisions. }\end{array}$ \\
\hline Study 1 & $\begin{array}{l}\text { Use content-enabled objects. When the length of the ECA's main narration about a location } \\
\text { must be reduced, use content-enabled objects placed on the background of the ECA. For } \\
\text { example, an ECA can give a basic narration about a location, and then "invite" players to } \\
\text { explore more about the location by tapping on the objects they are interested to learn more } \\
\text { about. }\end{array}$ \\
\hline Study 2 & $\begin{array}{l}\text { Enable the mobile guide application for QR-Code recognition. QR-Codes provide a cheap } \\
\text { alternative to more expensive and complex solutions such as GPS, for physical location tagging. }\end{array}$ \\
\hline Study 2, & $\begin{array}{l}\text { Give players control over the content and the visibility of the ECA. The minimum control } \\
\text { over narratives players expect is a repeat and pause functionality. Then, an option to control the } \\
\text { visibility of the ECA could benefit elderly players. We found evidence in study } 6 \text { that an ECA } \\
\text { could result into elderly participants feeling overwhelmed by the narratives. }\end{array}$ \\
\hline
\end{tabular}

\subsection{Interaction Design}

\begin{tabular}{|c|c|}
\hline Sources & \\
\hline Study 4 & $\begin{array}{l}\text { Prefer providing a random answer in Q\&A dialogues, than no answer at all. Although a } \\
\text { random answer may not be the right answer, it may contain fragments of the information the user } \\
\text { is seeking. This should in turn result in an increased user satisfaction as long as this process does } \\
\text { not take too much time. }\end{array}$ \\
\hline Study 4 & $\begin{array}{l}\text { When enhanced retention of cultural narratives is needed, motivate players to ask } \\
\text { questions about the narrative. We found that when players ask multiple questions in Q\&A, } \\
\text { their retention of cultural narratives increases. However, our study also highlighted the } \\
\text { importance of properly motivating participants to ask questions without becoming frustrated. A } \\
\text { way of providing positive motivation is to reward players with points (or other collectables) for } \\
\text { every question asked about the narratives. Points collected through Q\&A (and other activities) } \\
\text { can then be exchanged for gifts in the real world (e.g., a decorative plate from the castle of } \\
\text { Monemvasia). }\end{array}$ \\
\hline Study 6 & $\begin{array}{l}\text { Consider alternative types of ECA interruption messages for attracting attention. The } \\
\text { results of study } 6 \text { suggest that both serious and humorous messages worked in attracting } \\
\text { attention, but not in a player-friendly way. Hence, a combination of both humorous and serious } \\
\text { messages will most likely work more effectively than each of the strategies alone. This is } \\
\text { because mixing serious with humorous messages could result in diffusing any feelings of } \\
\text { discomfort while attracting an adequate level of attention. }\end{array}$ \\
\hline Study 6 & $\begin{array}{l}\text { Use a limited number of requests to attract the participants' attention to the narratives. } \\
\text { The ECA should request the participant's attention maximum } 3 \text { times. Then, it should rely on } \\
\text { alternative strategies to attract attention (e.g., to stop speaking for a few seconds). }\end{array}$ \\
\hline Study 6 & $\begin{array}{l}\text { Give participants time to become familiar with foreign names used in narratives. Players } \\
\text { should be given some time to become familiar with names that are foreign to their cultural } \\
\text { background (e.g., Monemvasia). A short training session with the guide system prior to the } \\
\text { beginning of the tour should enable players to become familiar with names that are unknown to } \\
\text { them. }\end{array}$ \\
\hline Study 3 & $\begin{array}{l}\text { Prefer images of landmarks for navigation over other methods. The literature suggests that } \\
\text { photographs of landmarks can be an effective method of navigation in mobile guide applications } \\
\text { [54]. Our experiences (see study 3) suggest that an ECA could help participants understanding } \\
\text { the underlying structure of a landmark through the use of relevant verbal and non-verbal } \\
\text { behaviours, which could result in improved navigation of routes in the real world. }\end{array}$ \\
\hline
\end{tabular}

\subsection{Simulation Design for Laboratory Testing}

\begin{tabular}{l|l}
\hline Sources & \multicolumn{1}{|c}{} \\
\hline Study 3 & $\begin{array}{l}\text { Use of high-definition sort-video clips over panoramas to simulate an outdoor environment. } \\
\text { When testing in the field is not possible, use video clips to simulate an environment, over } \\
\text { panoramas. Video-clips are easier and less expensive to produce. Furthermore, consider adding a } \\
\text { treadmill to give players the "feeling" of walking from one location to another. }\end{array}$ \\
\hline Study 1 & $\begin{array}{l}\text { Consider the use of more "natural" methods for player interaction with a simulated } \\
\text { environment. To avoid problems with players having difficulty synchronizing the mobile guide } \\
\text { application with the simulated environment, consider the use of more natural methods of } \\
\text { interaction (e.g., Microsoft Kinect [55]) }\end{array}$ \\
\hline
\end{tabular}




\section{Conclusions and Future Work}

The work, so far, shows the potential of the research framework to be used as a foundation for investigating the impact of gamified mobile guide applications using ECAs on the player's task performance and experience. Although, a part of the framework (i.e., Octalysis) has been used in the analysis of many commercial games for entertainment (e.g., [56]), the whole framework is not meant to be used in this way. Its focus is on games and gamified mobile applications where ECAs play an important role and enhanced learning performance is the desired output of the interaction process. Based on the framework, we also explain some controversial terms in gamified applications using ECAs, i.e., what cognitive accessibility, usability and player experience mean, at least in this context. Our studies showed us that an ECA capable of displaying some of the communication strategies (e.g., non-verbal behaviours to accompany linguistic information, etc.) found in the real-world human guidance scenario is not effective in enhancing the player's ability to retain cultural content. However, we found that an ECA can affect the quality of the player's experience while accomplishing the navigation and information tasks. Given the positive impact of ECAs on the player's experience, the lack of effectiveness could be attributed to the simulated mobile conditions in the laboratory. The use of immersive media (such as video and panoramic images) to simulate real environments has the drawback that it does not allow an authentic evaluation of the player's experience. It is possible that if players had experienced the mobile guides in the actual castle, the ECA would have elicited better navigation of routes and retention of cultural narratives than without it. We plan to use our framework to drive additional studies in the real castle of Monemvasia. A variable of particular interest is competition, and how a competitive task may impact the perception of a gamified mobile application and the player's task performance. It will be interesting to compare the results of a group with players that choose to compete in a "treasure hunt" style of taking a tour in the medieval castle with the results of a group with players that choose to take a regular tour in the same location.

\section{References}

[1] Doumanis, I. (2013): Evaluating Humanoid Embodied Conversational Agents in Mobile Guide Applications. Thesis (PhD). Middlesex University, London UK.

[2] Krüger, A., Baus, J., Heckmann, D., Kruppa, M., (2007): Adaptive Mobile Guides. The Adaptive Web, P. Brusilovsky, A. Kobsa \& W. Nejdl (Eds.), Springer, 2007, pp. $521-$ 549. http://dx.doi.org/10.1007/978-3-540-72079-9_17

[3] Wagner, D. and D. Schmalstieg (2006): Handheld Augmented Reality Displays. In Proceedings of the 2006 IEEE conference on Virtual Reality (VR2006) Alexandria, Virginia, USA, March 25 - 29, 2006, pp. 321 http://dx.doi.org/10.1109/vr.2006.67

[4] Apple Inc. (2015): iPad Tablet Device. Available at: http://www.apple.com/ipad/ [Accessed March 11 2015].

[5] Apple Inc. (2015): Siri Mobile Application. Available at: http://www.apple.com/ios/siri/ [Accessed March 11 2015].

[6] Waze Mobile (2015): Waze Mobile application. Available at: https://www.waze.com/.

[7] Mobee Inc. (2015): Mobee mobile application. Available at: http://www.mobeeapp.com/ [Accessed March 11 2015].

[8] Falke, C. (2014). Ghosts Games Wartburg. Available at: http://goo.gl/vd3fQ3 [Accessed March 11 2013].

[9] Next OS (2015): Virtual Assistance Denise. Available at: https://guile3d.com/en/ [Accessed March 11 2015].

[10] Dow, S., Mehta, M., Lausier, A., MacIntyre, B., Mateas, M., (2006): Initial Lessons from AR Façade, An Interactive Augmented Reality Drama. Proceedings of the International Conference on Advances in Computer Entertainment Technology, ACE 2006, Hollywood, California, USA, June 2006. http://dx.doi.org/10.1145/1178823.1178858

[11]Miyake, S., Ito, A., (2012): A Spoken Dialogue System Using Virtual Conversational Agent with Augmented Reality. Proceedings of Signal \& Information Processing Association Annual Summit and Conference (APSIPA ASC), 2012 Asia-Pacific December 3-6, 2012, pp. 1-4. 
[12]Cassell, J., \& Stone, M. (1999): Living hand to mouth: Psychological theories about speech and gesture in interactive dialogue systems. Proceedings of the AAAI Fall symposium '99, pp. 34-42.

[13]Reeves, B., \& Nass, C. (1996): The media equation: How people treat computers, television, and new media like real people and places. Cambridge University Press

[14]Lee, E.-J. \& Nass, C. (1998): Does the ethnicity of a computer agent matter? An experimental comparison of human-computer interaction and computer-mediated communication. Proceedings of the Workshop on Embedded Conversational Characters Conference. Lake Tahoe, CA, pp. 123-128

[15]Rickenberg, R., Reeves, B. (2000): The effects of Animated Characters on Anxiety, Task Performance, and Evaluations of User Interfaces. Proceedings of CHI 2000, The Hague, The Netherlands, Letters, 2(1), pp.49-56. http://dx.doi.org/10.1145/332040.332406

[16]Xiao, J. (2006): Empirical Studies on Embodied Conversational Agents. Thesis (PhD). Georgia Institute of Technology.

[17]GamEffective (2015): GamEffective Platform. Available at: http://www.gameffective.com/ products/overview/

[18]Mayer, R., \& Moreno R. (2002): Animation as an Aid to Multimedia Learning. $\begin{array}{lllll}\text { Educational } & \text { Psychology } & \text { Review } & \text { 14-1, }\end{array}$ http://dx.doi.org/10.1023/A:1013184611077

[19] Moreno, R., Mayer, R. (2000): Engaging Students in Active Learning: The Case for Personalized Multimedia Messages. Journal of Educational Psychology Vol 92(4), pp. 724-733. http://dx.doi.org/10.1037/0022-0663.92.4.724

[20]Baylor, A. L. and S. Kim (2008): The Effects of Agent Nonverbal Communication on Procedural and Attitudinal Learning Outcomes. International Conference on Intelligent Virtual Agents. Tokyo, Springer: pp. 208-214. http://dx.doi.org/10.1007/978-3-54085483-8_21

[21]Campos, H., Campos, J., Martinho, C., Paiva A. (2012): Virtual Agents in Conflict, 12th International Conference on Intelligent Virtual Agents (IVA 2012), September 20-22, 2012, Santa Cruz, CA, USA pp. 105-111. http://dx.doi.org/10.1007/978-3-642-331978_11. http://dx.doi.org/10.1007/978-3-642-33197-8_11

[22] Korre, D., (2015): Usability Engineering: Embodied Conversational Agents in Serious Games. Available at: http://goo.gl/TR72Kj

[23] Adams, R. and Langdon, P. (2003): SIMPLEX: a simple user check-model for Inclusive Design. Universal Access in HCI: Inclusive Design in the Information Society. Stephanidis, C. (Ed.). 4, pp. 13-17. Mahwah: NJ: Lawrence Erlbaum Associates.

[24] Adams, R. (2006): Decision and stress: cognition and e-accessibility in the information workplace. Universal Access in the Information Society, 5 (4). pp. 363-379. ISSN 16155297

[25] Sternberg, R. J. (Ed.). (2000). Handbook of intelligence. New York, USA: Cambridge University Press. http://dx.doi.org/10.1017/CBO9780511807947

[26]Chou, Y. (2015): Octalysis: Complete Gamification Framework. [Blog] Yu-Kai Chou \& Gamification. Available at: http://goo.gl/Qa1Vm2 [Accessed 29 March 2015].

[27] Adams, R. (2005): (R. G. Adams@ mdx.ac.uk) (25 June 2005). RE: Accessibility Evaluation based on Simplex. Personal email to I. Doumanis (idoumanis@mdx. ac.uk)

[28] ISO 9241-11 (1998). Ergonomics requirements for office work with visual display terminals (VDTs) - Part 11: Guidance on usability.

[29]Hollan, J., Hutchins, E., \& Kirsh, D. (2000): Distributed Cognition: Toward a New Foundation for Human Computer Interaction Research. ACM Trans. on ComputerHuman Interaction. 7 (2), pp. 174-196. http://dx.doi.org/10.1145/353485.353487

[30]Kaptelinin, Victor and Bonnie Nardi (1997): Activity Theory: Basic Concepts and $\begin{array}{lllll}\text { Applications. } & \text { Proceedings } & \text { of } & \text { CHI } & \text { 74-77. }\end{array}$ http://dx.doi.org/10.1145/1120212.1120321

[31]Craig, P., Roa-Seiler, N., Diaz, M. D, Rosano, L. R., (2013): The Role of Embodied Conversational Agents in Collaborative face to face Computer Supported Learning Games, Proceedings of the 25th International Conference on System Research, Informatics \& Cybernetics, Baden-Baden, Germany, July 29 -August 2, 2013.

[32]Katz, J. (2000). Up, up, down, down. Slashdot.org. Originally published November, 30, 2000. (http://slashdot.org/features/00/11/27/1648231.shtml)

[33] Prensky, M. (2000). Digital Game-Based Learning. New York: McGraw Hill. 
[34]Raessens, J.F.F. 2012: Homo Ludens 2.0. The Ludic Turn in Media Theory" Available at: http://goo.gl/38nPtz [Accessed March 11 2013]

[35]Cowell, A. J., Stanney, K. M. (2002): User demographics for embodiment customization. AAAI Fall Symposium on Personalized Agent. Cambridge, MA: American Association for Artificial Intelligence. November 15-17, 2002, pp. n1-n2.

[36]Chou, Y. (2015): Gamification Design: 4 Phases of a Player's Journey [Blog] Yu-Kai Chou \& Gamification. Available at: http://goo.gl/UYxuNN [Accessed 29 March 2015]

[37]Catrambone, R., Stasko, J., \& Xiao, J. (2002): Anthropomorphic agents as a user interface paradigm: Experimental findings and a framework for research. Proceedings of the 24th Annual Conference of the Cognitive Science Society, Fairfax, VA, August 2002, pp. 166171.

[38]Phan M. H., Jardina J. R., \& Hoyle W. S. (2012): Video Games: Males prefer violence while Females prefer social. Available at: http://goo.gl/z4rbvY [Accessed March 11 2013]

[39]Bartle, R. (1996). Hearts, clubs, diamonds, spades: players who suit MUDS. Available at: http://goo.gl/mp8oct [Accessed March 112015

[40] Gonzalez, Jr., P.A (2014): Race and Ethnicity in Video Games: A Reflection of Social Reality. Racism, hate speech and prejudice: a manifestation of social stereotypes. Available at: http://goo.gl/5EKi2q [Accessed March 11 2015].

[41] Gans, H. J. (1999). Popular culture and high culture: an analysis and evaluation of taste. New York: Basic Books.

[42]Levine, L. (1988) Highbrow/Lowbrow: the emergence of cultural hierarchy in America. Cambridge: Harvard University Press.

[43] Schilling, C. (2009): Art house video games. The Daily Telegraph (London). Available at: http://goo.gl/QUjUIV

[44] Griffiths, S. (2013): 'Granddad' Theft Auto: Middle-class, middle-aged parents are most likely to play violent crime game. Available at: http://goo.gl/BWpOC7

[45] Octalysis 2013. Enterprise Gamification Consultancy wiki [Online]. Available at: http://goo.gl/3khrVy [Accessed March 11 2015].

[46] Game Mechanics 2010. Gamification Wiki. [Online]. Available at http://goo.gl/ yTHT81

[47]Likert, R. (1932): A Technique for the Measurement of Attitudes. In: Archives of Psychology 140, p.55.

[48]Doumanis I., and Smith S (2014): Evaluating the impact of Embodied Conversational Agents attentional behaviours on user retention of cultural content in a simulated mobile environment. Proceedings of 16th ACM International Conference on Multimodal Interaction (ICMI 2014), November 16th, 2014, Istanbul, Turkey.

[49]Doumanis I., Serengul, S., "Evaluating an Intelligent Q\&A system for Mobile Cultural Learning" In Journal of EAI Endorsed Transactions on Future Intelligent Educational Environments, Vol.1, Issue 2, June 2015, pp. 1-11.

[50]Doumanis, I., Serengul, S., (2013): An empirical study on the effects of Embodied Conversational Agents (ECAs) on user retention performance and perception in a simulated mobile environment. Proceedings of the $9^{\text {th }}$ International Conference Intelligent Environments (IE'13) 16-17 July 2013, Athens Greece, pp. 431-442.

[51]Doumanis, I., Serengul, S., (2013): Evaluating the Impact of ECAs on User navigation performance and perception. Proceedings of the 13th International Conference on Intelligent Virtual Agents (IVA 2013) 29-31 August 2013, Edinburgh, UK, pp. 474-475.

[52]Virtual People Factory (VPF) (2013): Available at: http://goo.gl/MKA1W3 [Accessed March 11 2013].

[53]MacDorman, K. F., \& Ishiguro, H. (2006): The uncanny advantage of using androids in social and cognitive science research. Interaction Studies, Volume 7, Issue 3(2006), pp. 297-337. http://dx.doi.org/10.1075/is.7.3.03mac

[54] Hile, H., Vedantham, R., Liu, A., Gelfand, N., Cuellar, G., Grzeszczuk, R., Borriello, G. (2008): "Landmark-Based Pedestrian Navigation from Collections of Geotagged Photos". In: Proceedings of ACM International Conference on Mobile and Ubiquitous Multimedia (MUM 2008), December 3 - 5 2008, Umea, Sweden, pp.145-152. http://dx.doi.org/10.1145/1543137.1543167

[55] Microsoft Inc. (2015): Kinect for Windows. Available at: https://www.microsoft.com/enus/kinectforwindows/ [Accessed March 11 2015]. 
pag. 40

[56] Chou, Y. (2015): Game Design Analysis of Diablo II through Level II Octalysis [Blog] Yu-Kai Chou \& Gamification. Available at: http://goo.gl/5dlMP7 [Accessed 29 March 2015]. 